\title{
Neuropathological findings in Miller Fisher syndrome
}

\author{
MS PHILLIPS, S STEWART, JR ANDERSON \\ From the Departments of Medicine and Pathology, Peterborough District Hospital, Peterborough, \\ Cambridgeshire, and Department of Pathology, Addenbrooke's Hospital, Cambridge, UK
}

SUMMARY The neuropathological findings in a fatal case of Miller Fisher syndrome are described. The demyelinating peripheral neuropathy and normal appearance of the central nervous system that were observed support the inclusion of the syndrome within the spectrum of acute inflammatory polyneuropathy.

The syndrome of acute ophthalmoplegia, ataxia and areflexia, described by Fisher in $1956^{1}$ is generally agreed to be a variant of acute inflammatory polyneuropathy or Guillain-Barré syndrome. It is rare and usually benign. As a result, no detailed neuropathological studies have been reported. Its inclusion within the spectrum of acute inflammatory polyneuropathy was initially based on the clinical findings and cerebrospinal fluid laboratory observations $^{2}$ later supported by nerve conduction studies and electromyography, which have confirmed a peripheral neuropathy, probably of a demyelinating type. ${ }^{3}$

Doubt has existed as to whether all the manifestations of the syndrome could be attributed solely to a disorder of peripheral nerves. In particular, the often profound degree of ataxia in relation to the slight sensory and motor deficit has raised the question of cerebellar involvement. Also some of the abnormalities of eye movement suggest a central rather than peripheral lesion. ${ }^{4}$ An alternative explanation of "brain stem encephalitis" has been given and recently re-emphasised. ${ }^{\circ}$

We report the neuropathological findings in a recently observed case and consider how they might influence these doubts and clarify the position of the syndrome within the classification of acute inflammatory polyneuropathy.

\section{Case report}

HC, a 67-year-old English woman, was admitted to hospital on the 20th January 1982 with a three day history of difficulty with balance, loss of control of the limbs, inter-

Address for reprint requests: MS Phillips, Addenbrooke's Hospital, Cambridgeshire, CB2 2QQ, UK.

Received 30 August 1983 and in revised form 7 December 1983. Accepted 8 December 1983 mittent double vision and unpleasant sensations in her hands and feet.

Three months prior to admission, digoxin $0.25 \mathrm{mgms}$ daily and Navidrex $\mathrm{K}$ two tablets daily had been prescribed for ankle swelling. One week prior to admission, she had suffered a twenty four hour period of nausea, vomiting and diarrhoea, which had settled spontaneously.

She smoked fifteen cigarettes a day and admitted a modest alcohol intake. There was no other personal medical history of note but two sisters were insulin-dependent diabetics.

On examination she was alert and orientated. Nasal speech was noted. The fundi were normal but the pupils were round, equal and dilated to $8 \mathrm{~mm}$ diameter. There was no reaction to light nor accommodation in either eye and a general decrease in the range of external ocular movements, with particular restriction of abduction of both eyes. Within twenty four hours, a total bilateral external ophthalmoplegia developed. Partial ptosis and moderate facial weakness were noted bilaterally. Elevation of the soft palate was symmetrical but reduced and the cough was weak. The limbs were wildly inco-ordinate and badly bruised. In the arms, tone was normal, but a flapping irregular tremor of the outstretched hands and fingers, together with a symmetrical, predominantly proximal weakness was observed. Joint position sense was absent to the elbow on the right and to the wrist on the left, whilst a diminished appreciation of light touch and pin-prick was found on all the fingers. Tone in the legs was normal with a symmetrical proximal weakness, but normal power distally. Joint position sense was impaired in both great toes but other sensory modalities were normal. There was complete areflexia; plantar responses were flexor. She was quite unable to stand or even sit erect. The general examination was unremarkable (blood pressure 180/100 $\mathrm{mm} \mathrm{Hg}$ ). A clinical diagnosis of Miller Fisher syndrome was made.

Electrophysiological studies were performed six days after admission. Electromyographic exploration of right abductor hallucis and extensor digitorum brevis showed fibrillation at rest and discrete volitional activity. Conduction velocity was $31 \mathrm{~m} / \mathrm{s}$ down the lateral popliteal nerve and $29 \mathrm{~m} / \mathrm{s}$ down the medial popliteal nerve. Exploration 
of left abductor hallucis and extensor digitorum brevis again showed fibrillation at rest with discrete volitional activity. Conduction velocity was $30 \mathrm{~m} / \mathrm{s}$ down the lateral popliteal nerve and $31 \mathrm{~m} / \mathrm{s}$ down the medial popliteal nerve. Sural sensory action potentiais were absent in both legs.

Lumbar puncture nine days after admission revealed clear colourless cerebrospinal fluid with an opening pressure of $8 \mathrm{cms}$ of CSF. The protein content was $1.4 \mathrm{~g} / \mathrm{l}$. It contained 3 mononuclear cells and 24 erythrocytes per $\mathrm{mm}^{3}$ and $4 \mathrm{mmol} / \mathrm{l}$ of glucose.

The chest radiograph demonstrated an elevated left hemidiaphragm consistent with a left phrenic nerve paralysis. Full blood count was unremarkable and fasting blood glucose normal. Screening tests for syphilis (TPHA and VDRL) and the Paul Bunnell test were negative, but serological testing revealed a compliment fixing titre to influenza $A$ of 1 in 160, indicating recent infection. Porphyrins were not detected in the urine. Neither Tensilon nor Parentrovite administered intravenously altered the appearance of the eyes.

The clinical picture remained unaltered for three weeks. Abnormalities of sweating, spontaneous fluctuations of pulse and blood pressure, postural hypotension and ventilatory difficulty were not observed. The vital capacity was low at $1 \cdot 1$ litres (predicted $2 \cdot 3$ litres) but it remained constant. Regular physiotherapy seemed successful in keeping the chest free of infection and no dysphagia was observed. No respiratory abnormalities were noted during sleep, but she died unexpectedly, at night, twenty seven days after admission.

\section{Pathological findings}

A post mortem examination was performed approximately twenty seven hours after death and revealed a terminal bronchopneumonia. The coronary arteries were moderately atherosclerotic. There was a severe haemorrhagic cystitis but no evidence of ascending infection involving the kidneys. No macroscopic abnormality was found in the central or peripheral nervous system.

Microscopic examination of the spinal nerve roots and peripheral nerves revealed patchy, but extensive recent segmental demyelination (fig 1). The changes appeared to be of approximately the same age in all the nerves and consisted of swollen and fragmented myelin sheaths with a few associated foamy macrophages and lymphocytes. The axons were preserved, in general, but some showed swelling and tortuosity in the demyelinated region. There was scanty perivascular chronic inflammatory cell infiltration, consisting mainly of small lymphocytes in the larger nerve trunks. Sections through the spinal cord showed involvement of both motor and sensory nerve roots (fig 2 ). Examination of the cranial nerves revealed the same patchy demyelination and mild cellular infiltration involving the 7 th, 10th and 11 th nerves. The optic nerves appeared normally myelinated. The distal protions of the 3rd, 4th and 6th cranial nerves were not available for examination but no histological abnormality of their more proximal portions was found. The brain stem and cerebellum were histologically normal.

The deltoid and gastrocnemius muscles of both sides showed moderate fibre atrophy, these fibres showing angu-

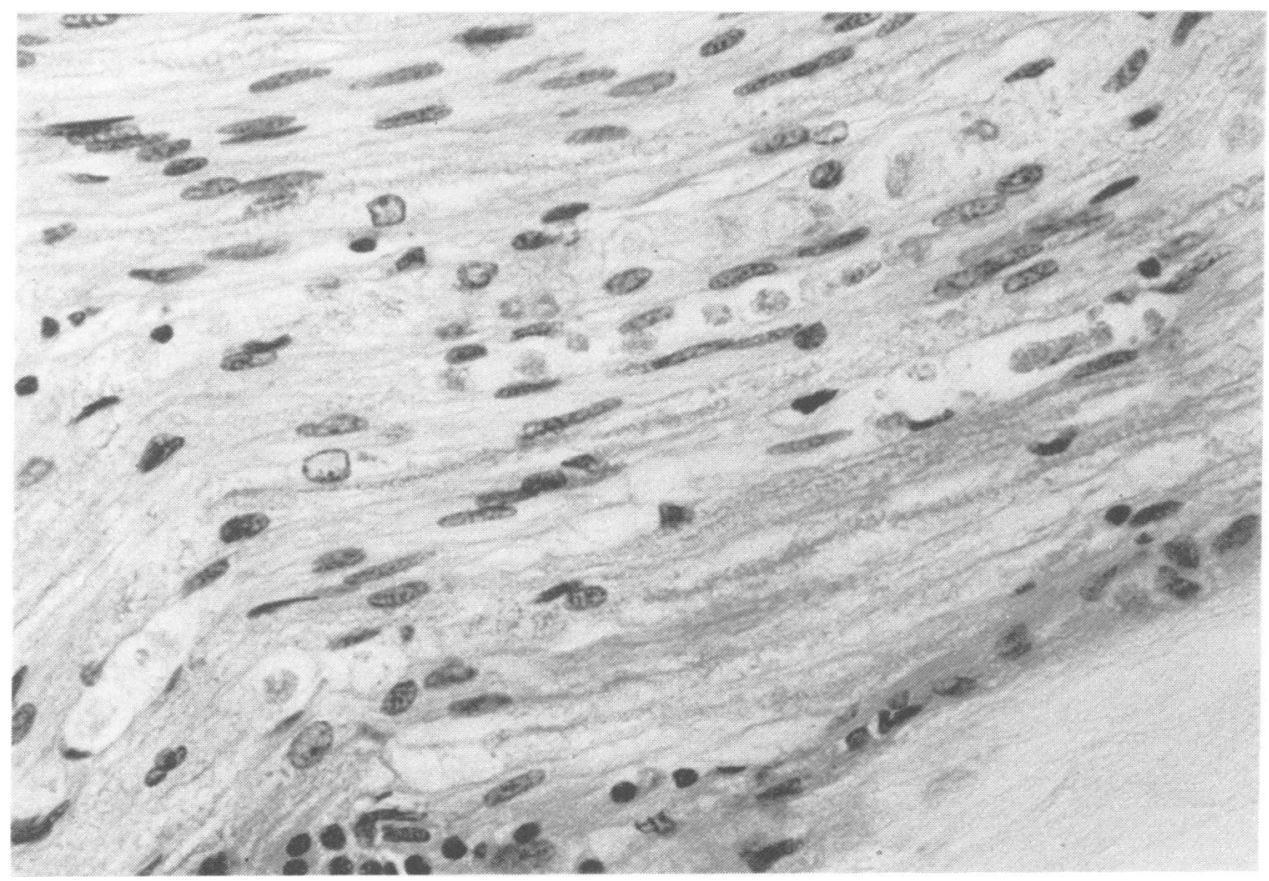

Fig 1 Peripheral (sciatic) nerve showing extensive demyelination associated with some foamy macrophages and scanty lymphocytes. (Solochrome cyanin stain for myelin. $\times 126$ ) 


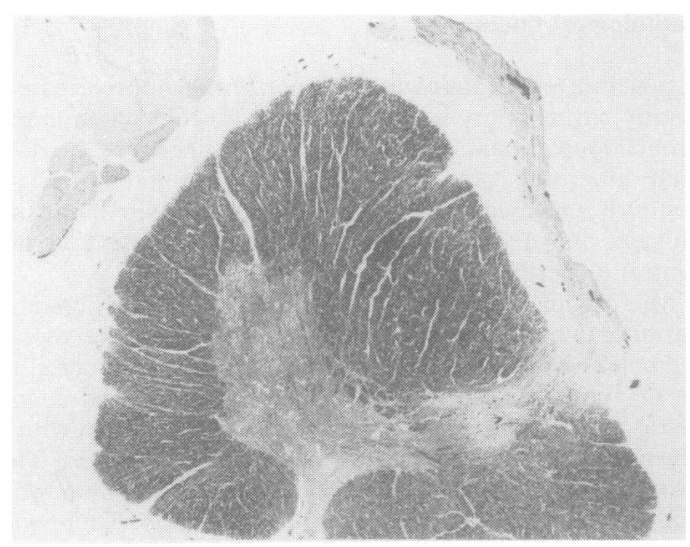

Fig 2 Transverse section of spinal cord showing pallor of demyelinated motor and sensory nerve roots. (Solochrome cyanin stain for myelin. $\times 8$ )

lar outlines. Grouped atrophy was present in the most abnormal areas. The ATP-ase reaction on frozen sections of muscle showed both type 1 and type 2 fibres were involved with type 1 fibres most severely affected.

Electron microscopy of the affected spinal nerve roots and peripheral nerves revealed more prevalent myelin destruction than the paraffin sections had suggested. Lymphocytes and macrophages but no plasma cells were seen in the areas of myelin breakdown. The most conspicuous change was that of vesicular dissolution of myelin, predominantly intracellular. Splitting of the myelin sheaths unrelated to macrophages was also observed and associated with intramyelinic oedema. Many demyelinated axons were seen (figs 3 and 4), some of these surrounded by pale Schwann cell cytoplasm. There was no definite evidence of remyelination. Viral culture of the brain was negative.

\section{Discussion}

Light and electron microscopy showed features well

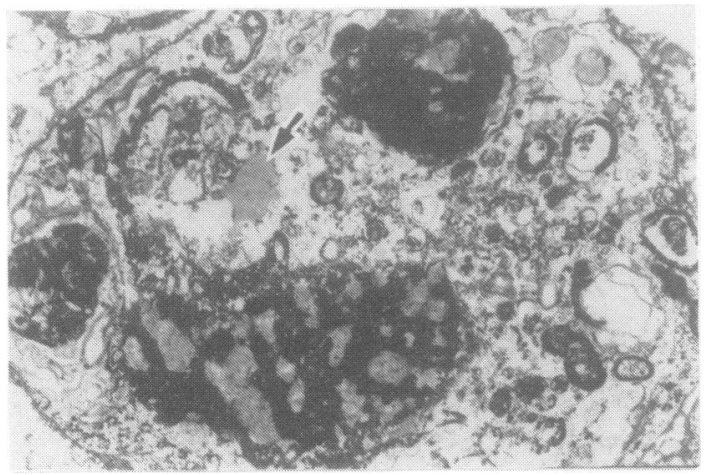

Fig 3 Shrunken axon (arrow) surrounded by myelin sheath showing vesicular breakdown. Sciatic nerve. (Electron micrograph. $\times 2172$ )

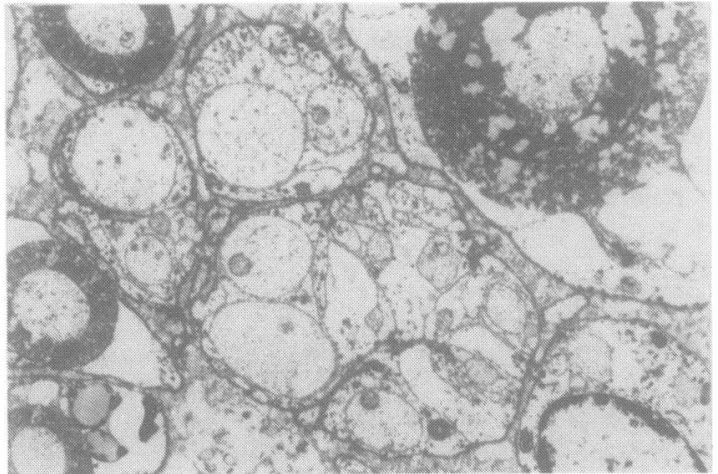

Fig 4 Completely demyelinated axons in Schwann cell cytoplasm. Sciatic nerve. (Electron micrograph. $\times 2830$ )

described in acute inflammatory polyneuropathy..$^{7-9}$ The prevalence of vesicular myelin dissolution has been noted to be higher in necropsy material than in tissue taken during life but has been reported to be mainly extracellular. ${ }^{1011}$ Some of the vesicular breakdown in our study may indeed represent post mortem change but its association with lymphocytes and macrophages make it unlikely that it is all artefactual. It is possible that some of the myelin splitting observed in the absence of inflammatory cells represent myelinolysis occurring after death. In fresh tissues from acute inflammatory polyneuropathy obtained by sural nerve biopsy, phagocytosis of structurally normal myelin lamellae by insinuated macrophage processes is commonly observed. ${ }^{12}$ This was infrequently seen in our material which may be due to the fact that this is a relatively early feature of myelin breakdown and that our patient died thirty days after the onset of neurological symptoms. The third type of myelin change observed in acute idiopathic polyneuropathy is myelin bubble formation, thought to be a residual alteration in the nerves; it was not identified by us. ${ }^{9}$

The absence of any evidence of remyelination is not unexpected in view of the lack of clinical improvement before death. The skeletal muscle changes were quite typical of recent denervation ${ }^{13}$ and showed no features of renervation.

Death was due to bronchopneumonia, which although not always clinically apparent is the usual cause of death in inflammatory polyneuropathy. ${ }^{8}$

In his description of this syndrome, ${ }^{1}$ Fisher pointed out that several features, notably the degree and symmetry of the ophthalmoplegia, comparatively mild ptosis, relative sparing of downward gaze and cerebellar-type ataxia, suggested a central lesion. Also, the degree of sensory loss seemed mild if the areflexia were due to peripheral neuropathy. 
Nevertheless, he concluded that the clinical signs he observed were due to a Guillain-Barré like process affecting the peripheral nerves.

This conclusion has been generally accepted and support for a peripheral neuropathy has been obtained from nerve conduction and electromyographic studies. ${ }^{3}$ However, doubt about a central lesion has continued and recently a series of eighteen patients all with many of the features of this syndrome was reported under the title "Brain stem encephalitis and the syndrome of Miller Fisher". 6 CT scans of the brain in three out of the nine patients in which they were performed demonstrated low density abnormalities in the brain stem. Post mortem examination of the corresponding area in one patient showed changes interpreted as evidence of encephalitis.

It has been remarked that a brain stem lesion capable of causing all the features of the Miller Fisher syndrome would have to be remarkably selective in which structures it affected and which it spared. ${ }^{14}$ Such a lesion seems unlikely, and there is no convincingly demonstrated proof of its existence.

It was not possible to examine the distal portions of the 3rd, 4th and 6th cranial nerves in our patient, and the proximal portions were normal. Therefore we cannot prove a peripheral cause for the ophthalmoplegia. However we suggest that the pathological findings of extensive demyelination of both peripheral and cranial nerves, without pathological changes in the brain and spinal cord are characteristic of a demyelinating peripheral neuropathy of Guillain-Barré type, and thus support the inclusion of Miller Fisher syndrome within the spectrum of acute inflammatory polyneuropathy.

We thank Dr D Guttmann for his permission to report the details of his patient, Dr N Williams for performing the electrophysiological studies and Ms
M Taylor for technical assistance with the neuropathology.

\section{References}

' Fisher, Miller. An unusual variant of acute idiopathic polyneuritis (syndrome of ophthalmoplegia, ataxia and areflexia). $N$ Engl $J$ Med 1956;255;2:57-65.

${ }^{2}$ Asbury AK. Diagnostic considerations in Guillain-Barré syndrome. Ann Neurol 1981;9 (suppl):1-5.

${ }^{3}$ Guilloff RJ. Peripheral nerve conduction in Miller Fisher syndrome. J Neurol Neurosurg Psychiatry 1977; 40:801-7.

${ }^{4}$ Meienberg O, Ryffel E. Supranuclear Eye Movement Disorders in Fisher's Syndrome of ophthalmoplegia, ataxia and areflexia. Arch Neurol 1983;40:402-5.

${ }^{5}$ Bickerstaff ER. Brain stem encephalitis. Further observations on a grave syndrome with benign prognosis. Br Med J 1957;1:1384-7.

- Al-Din AN, Anderson M, Bickerstaff BR, Harvey I. Brain stem encephalitis and the syndrome of Miller Fisher. A Clinical Study. Brain 1982;105:481-95.

' Prineas JW. Pathology of the Guillain-Barré syndrome. Ann Neurol 1981;9 (suppl):6-19.

${ }^{8}$ Haymaker W, Kernohan JW. The Landry-GuillainBarré syndrome. A clinico-pathologic report of fifty fatal cases and a critique of the literature. Medicine (Baltimore) 1949;28:59-141.

${ }^{9}$ Prineas JW. Acute idiopathic polyneuritis. An Electron Microscope Study. Lab Invest 1972;26:133-47.

${ }^{10} \mathrm{Lui} \mathrm{HM}$. Ultrasound of remyelination of peripheral nerves in Landry-Guillain-Barré syndrome. Acka Neuropathol (Berl) 1970;16:262-5.

"Wisneuwski A, et al. Landry-Guillain-Barré syndrome. A primary demyelinating disease. Arch Neurol 1969; 21:269-76.

${ }^{12}$ Prineas JW, McLeod JG. Chronic relapsing polyneuritis. J Neurol Sci 1976;77:427-58.

${ }^{13}$ Harriman DGF. In: Blackwood W, Corsellis JAN, eds. Greenfield's Neuropathology 3rd Ed. Chap. 19, London. E Arnold, 1976.

${ }^{14}$ Ropper AH. The CNS in Guillain-Barré Syndrome (Editorial). Arch Neurol 1983;40:397-8. 